\title{
No evidence of a correlation between lumbar spinal subtypes and intervertebral disc degeneration among asymptomatic middle-aged and aged patients
}

\author{
BEN WANG ${ }^{1,2}$, YIFENG SHI $^{1,2}$, BODA CHEN $^{1,2}$, ZHENXUAN SHAO $^{1,2}$, LIJIE CHEN $^{3}$, \\ JIALIANG LIN ${ }^{1,2}$, CHONGAN HUANG ${ }^{1,2}$, YINGZHAO YAN ${ }^{4}$ and XIANGYANG WANG ${ }^{1,2}$ \\ ${ }^{1}$ Department of Orthopaedics, The Second Affiliated Hospital and Yuying Children's Hospital of Wenzhou Medical University; \\ ${ }^{2}$ Key Laboratory of Orthopaedics of Zhejiang Province, Wenzhou, Zhejiang 325027; ${ }^{3}$ Department of Surgical Oncology, \\ Taizhou Hospital of Wenzhou Medical University, Taizhou, Zhejiang 317000; ${ }^{4}$ Department of Orthopaedics, \\ Zhejiang Hospital, Hangzhou, Zhejiang 310012, P.R. China
}

Received June 1, 2019; Accepted February 18, 2020

DOI: $10.3892 /$ etm.2020.9055

\begin{abstract}
The aim of the present study was to identify whether lumbar spinal subtypes (LSS) were associated with lumbar disc degeneration (LDD) among asymptomatic middle-aged and aged subjects. A cohort of 158 asymptomatic Chinese adults aged $>40$ years was recruited and 97 volunteers that met the inclusion criteria with complete information available were selected for inclusion. According to spinal morphology, volunteers were divided into four subtypes based on the classification of Roussouly. After baseline information was collected and spinopelvic parameters were measured, the data were compared among the four groups. According to the Pfirrmann classification, the degree of LDD was evaluated at each level on the MRI. For grades I-V, LDD at each level was effectively compared. Each of the four LSS from I to IV according to Roussouly classification from types I to IV were comprised of 25 (25.8\%), 19 (19.6\%), 38 (39.2\%) and $15(15.5 \%)$ of volunteers, respectively. Lumbar lordosis, sacral slope and pelvic incidence were significantly different among the four sub-types $(\mathrm{P}<0.001$ for each), but no difference in pelvic tilt was observed $(\mathrm{P}=0.21)$. From types I to IV LSS, the proportion of disc degeneration was found to be $44,52,50$ and $48 \%$, respectively, which exhibited no statistically significant difference among LSS. No correlation between LSS and intervertebral
\end{abstract}

Correspondence to: Dr Xiangyang Wang, Department of Orthopaedics, The Second Affiliated Hospital and Yuying Children's Hospital of Wenzhou Medical University, 109 Xueyuan Xi Road, Wenzhou, Zhejiang 325027, P.R. China

E-mail: xiangyangwang@wmu.edu.cn

Dr Yingzhao Yan, Department of Orthopaedics, Zhejiang Hospital, 1229 Gudun Road, Hangzhou, Zhejiang 310012, P.R. China E-mail: yingzhaoyan99@163.com

Key words: lumbar spinal subtypes, spinopelvic parameters, disc degeneration, risk factors disc degeneration was obtained among the asymptomatic middle-aged and aged subjects. The present study provides a reference for spinal surgery and indicated that additional risk factors should be assessed in the asymptomatic population of this age group, particularly in terms of differentially expressed genes.

\section{Introduction}

Lower back pain, which is one of the most common condition, not only causes considerable disability and compromised quality of life but also places a burden on the family of the patient affected (1,2). Lumbar disc degeneration (LDD), as the pathogenic cause of discogenic pain, has previously been correlated with lower back pain (3). The proportion of LDD still remains high in the asymptomatic population $(4,5)$. The development of LDD has previously been described as a progressive process from mild to severe. Considering the essential prevention and evaluation of LDD, it is increasingly important to investigate the potential risk factors of LDD in asymptomatic individuals.

A variety of patient-specific internal and external factors contribute to the initiation and progression of LDD, including biomechanics, heritability, environmental factors, systemic diseases and smoking (3,6-10). At present, as a possible causative mechanism, biomechanical factors and sagittal alignment are used to influence intervertebral disc degeneration (11). Lumbosacral morphology has also been considered to serve a potential role in the degree of LDD and herniation (12). However, these aforementioned studies are aimed at symptomatic individuals with lower back pain or incapacitating symptoms. Whether biomechanical factors serve a major role in LDD in the asymptomatic population has remained to be determined and the influence of spinal morphology, including lumbar spinal subtypes (LSS) on disc degeneration, also remains controversial.

To systematically describe the normal sagittal alignment of the lumbar spine in asymptomatic young adults, Roussouly et al (13) proposed a four-point classification system 
based on lumbar and pelvic parameters. The lumbar postural subtypes were classified by sacral slope (SS) and spinal morphology (13). Subsequently, according to the shape of the lumbar spine, Roussouly and Pinheiro-Franco (14) proposed a different process of degeneration. The classification is as follows: Type I: The SS is $<35^{\circ}$ and the center of the L5 vertebral body is located at the apex of lumbar lordosis (LL). The lower or upper arc angle is minimal and the inflexion point is lower or posterior. Due to the L4-5 hyperextension, it may induce a nutcracker L5 spondylolysis. Type II: The SS is $<35^{\circ}$ and the base of the L4 vertebral body is located in the apex of lumbar lordosis. The LL arc is flat. Type II has a high risk of early disc herniation. Type III: The SS is between $35^{\circ}$ and $45^{\circ}$. Type III: An average shape that does not hold characteristics for a specific degeneration. Type IV: The SS is $>45^{\circ}$. Type IV: Retains the lordosis curvature, which may result in a degenerative L4/L5 spondylolisthesis. To the best of our knowledge, the association between LSS and LDD has only been investigated in young asymptomatic adults between 20 to 40 years of age (4), while, the correlation in asymptomatic middle-aged and aged adults has remained to be determined. There are two major reasons. First, in this specific asymptomatic population, the natural degeneration of intervertebral disc was universally occurring; thus, investigating this population was considered to be of high significance. Furthermore, asymptomatic middle-aged and aged individuals were difficult to be recruited. These are the reasons for the remaining lack of relevant studies in this population. Based on the above reasons, this specific population was examined in the present study.

The aim of the present study was to identify whether lumbar subtypes and spinopelvic parameters are associated with LDD in middle-aged and aged individuals. In the present study, it was assumed that lumbar sagittal alignment and LSS do not have the power to result in level-specific predilection for LDD; therefore, it was expected that no difference would be observed.

\section{Materials and methods}

Study population. The present study was a single-center, retrospective data analysis that aimed to investigate the correlation between LSS and LDD in middle and old-aged asymptomatic volunteers. Following approval by the institutional review board of The Second Affiliated Hospital and Yuying Children's Hospital of Wenzhou Medical University (Wenzhou, China), a cohort of 158 asymptomatic Chinese adults aged $>40$ years encountered between May 2016 and November 2018 at the Second Affiliated Hospital and Yuying Children's Hospital of Wenzhou Medical University (Wenzhou, China) was recruited. All volunteers provided written informed consent prior to enrollment.

The inclusion criteria were as follows: Volunteers aged $>40$ years, with available standing lumbar plain film radiographs. Once these volunteers met the inclusion criteria, an MRI of the lumbar spine was performed using a 3T MR scanner (Discovery 750; GE Healthcare). The exclusion criteria were as follows (15): i) Lameness or unequal length of the lower limbs; ii) significant scoliosis (Cobb angle $>10^{\circ}$ in the coronal position) (16); iii) a history of trauma of the lower extremities, pelvis or spine; iv) a history of hip or knee arthroplasty

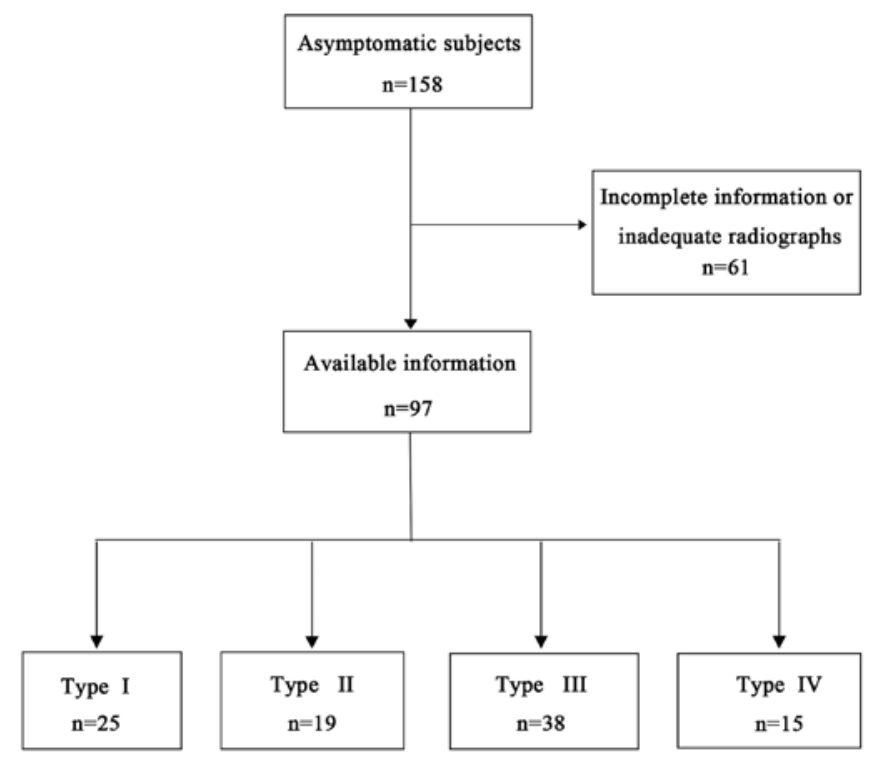

Figure 1. Flow-chart of the selection for different lumbar spinal subtypes among asymptomatic adults.

and/or spinal, pelvic or lower-limb surgery; v) complaints of back pain, neck pain or limb numbness caused by degenerative diseases of the spine, including disc herniation, spinal canal stenosis and lumbar spondylolisthesis; vi) strabismus or torticollis affecting balance; vii) a history of neuromuscular disorders or congenital abnormalities; viii) pregnancy or preparation for pregnancy.

Of the 158 subjects, 61 volunteers who had incomplete information or inadequate radiographs available, or those who met the exclusion criteria were excluded. Subsequently, a total of 97 asymptomatic volunteers were included in the present study, and baseline information was recorded, including age, weight, height and BMI. Finally, cases were classified into four groups according to LSS after carefully evaluating standing lumbar plain film radiographs: Type I, $\mathrm{n}=25$; Type II, $\mathrm{n}=19$; Type III, n=38 and Type IV, n=15 (Figs. 1 and 2).

Radiographic analysis and data collection. Lumbar spinal standing anteroposterior and lateral radiographs were acquired for all volunteers with their arms in the fists-on-clavicles position (17). The radiographs were examined by a spine surgeon who had independently reviewed hundreds of images previously. Parameters collected from plain film radiographs included LL, SS, pelvic tilt (PT) and pelvic incidence (PI). The LL is defined as the subtended angle between the upper endplate of L1 and the superior end plate of S1. The SS is defined as the angle between the horizontal and the upper sacral endplate. The PT is defined as the angle between the vertical and the line through the midpoint of the sacral plate to femoral head axis. The PI is defined as the angle perpendicular to the upper sacral endplate at its midpoint and the line connecting this point to the femoral head axis.

The degree of each intervertebral disc degeneration, which was based on the classification of Pfirrmann, was evaluated by two spinal surgeons (CAH and YZY) with $>5$ years of experience using MRI (Fig. 3) (18). Controversial discs were then subsequently presented to a third spinal surgeon 

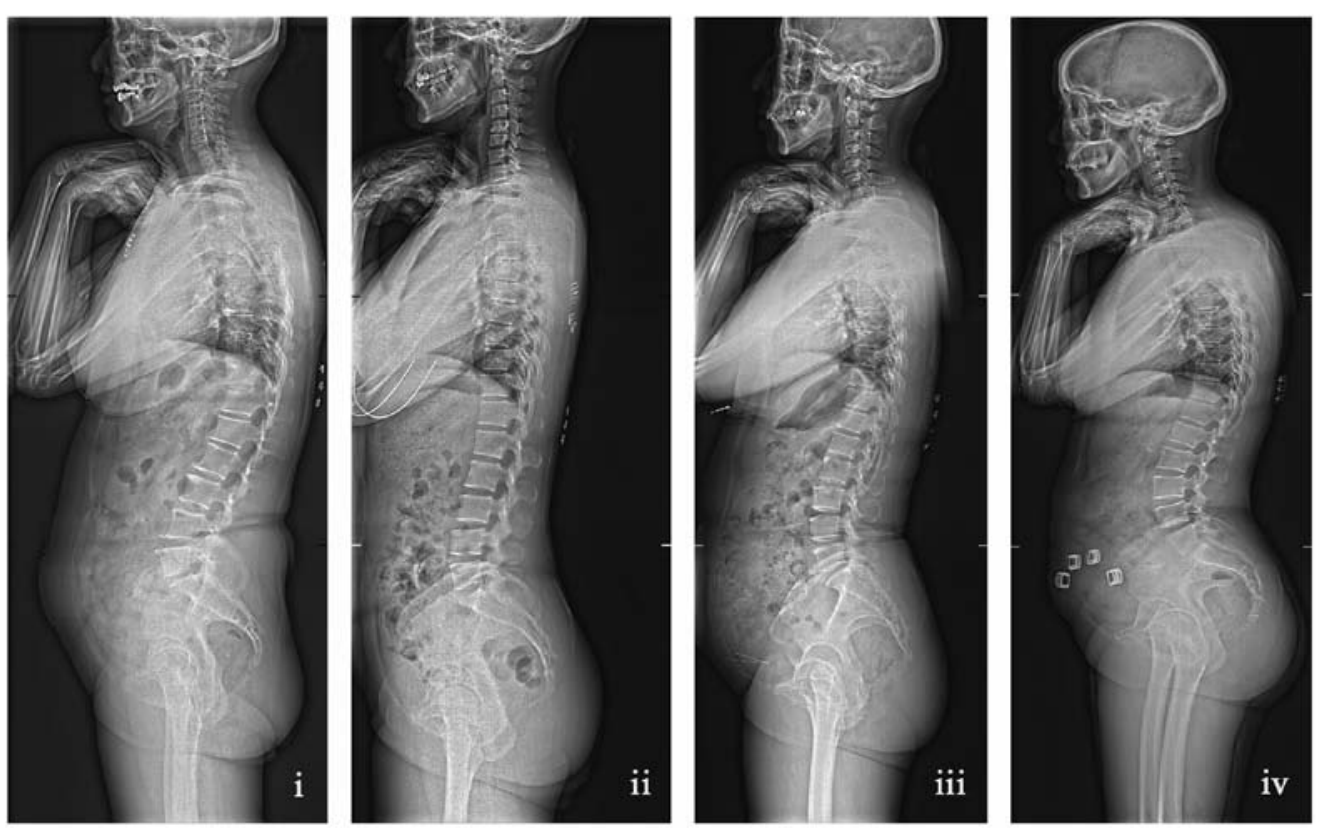

Figure 2. Representative images of the 4 types of lumbar spinal subtypes based on standing lateral radiographs. (i) Roussouly Type I; (ii) Roussouly Type II; (iii) Roussouly Type III; (iv) Roussouly Type IV.

(XYW) who provided the final evaluation. According to the Pfirrmann grades (I-V) (4), discs were then categorized as two groups: Non-degenerated (Pfirrmann $\leq \mathrm{II})$ and degenerated (Pfirrmann $\geq$ III).

Statistical analysis. Data in the present study were presented as either the mean \pm standard deviation or median (interquartile range). Following distribution analysis according to the Shapiro-Wilk test, baseline information and spinopelvic parameters were compared using a Kruskal-Wallis, $\chi^{2}$ test or one-way analysis of variance (ANOVA) to compare the variance of observed values among the LSS types, including values for age, gender, body mass index (BMI), SS, PI, PT and LL. The frequency of LDD among the four LSS types were compared using a $\chi^{2}$ or Fisher's exact test. All statistical analyses were performed using SPSS 22.0 (IBM Corp.). $\mathrm{P}<0.05$ was considered to indicate a statistically significant difference.

\section{Results}

Baseline data and measurement of parameters. For type I-IV LSS, there were differences in the proportion of volunteers, where the number of individuals in the type III subgroup was largest. As presented in Fig. 1, 25 (25.78\%), 19 (19.59\%), 38 (39.18\%) and 15 subjects $(15.46 \%)$ were included in group I-IV, respectively. All baseline data and certain radiographic parameters, including PI and PT, demonstrated a normal distribution, which represented real-valued random variables and they were compared between groups using one-way ANOVA. SS and LL were compared using a Kruskal-Wallis test due to data having a skewed distribution. As presented in Table I, baseline data were approximately equal for all four groups and exhibited no statistically significant difference, including for age, gender, body weight, body height and BMI (all P>0.05). In terms of the lumbar spine sagittal alignment, the values in type I were

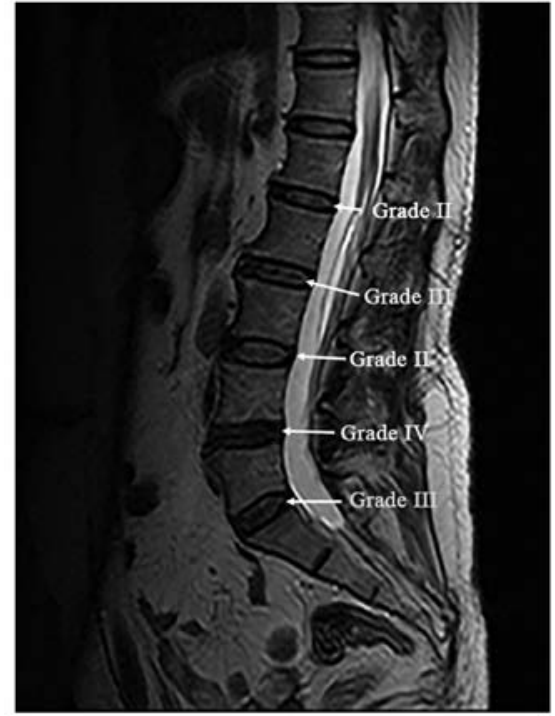

Figure 3. Different Pfirrmann grades of lumbar disc degeneration by level indicated in the MRI of a representative case.

as follows: SS, $29.4^{\circ}\left(25.6 ; 33.5^{\circ}\right)$; PI, $39.1 \pm 5.6^{\circ}$; PT, 9.9 $\pm 5.0^{\circ}$; $\mathrm{LL}, 38.5^{\circ}\left(31.9,46.1^{\circ}\right)$. The average values in type II were as follows: SS, $31.8^{\circ}\left(28.8 ; 34.3^{\circ}\right)$; PI, $41.1 \pm 5.5^{\circ}$; PT, $10.0 \pm 4.5^{\circ}$; LL, $44.5^{\circ}\left(35.7 ; 48.8^{\circ}\right)$. Average values in type III were as follows: SS, $39.0^{\circ}\left(37.2 ; 41.0^{\circ}\right)$; PI, $49.8 \pm 8.2^{\circ}$; PT, $10.8 \pm 7.3^{\circ}$; LL, 53.2 $\left(47.7 ; 56.5^{\circ}\right)$. Average values in type IV were as follows: SS, $45.9^{\circ}$ $\left(45.2 ; 48.3^{\circ}\right)$; PI, $58.4 \pm 7.3^{\circ}$; PT, $11.4 \pm 5.2^{\circ}$; LL, $63.8^{\circ}\left(60.0 ; 68.7^{\circ}\right)$. Statistically significant differences among the four LSS groups were observed for SS, PI and LL ( $\mathrm{P}<0.001$ for each), but no significant difference was observed for $\mathrm{PT}(\mathrm{P}=0.21)$.

Assessment of $L D D$. To assess the correlation between LSS and LDD, the degree of disc degeneration was assessed 
Table I. Clinicopathological characteristics of patients by Roussouly type.

\begin{tabular}{|c|c|c|c|c|c|}
\hline Variable & Type I (n=25) & Type II (n=19) & Type III (n=38) & Type IV $(n=15)$ & P-value \\
\hline Sex (male/female) & $4 / 21$ & $10 / 9$ & $13 / 25$ & $5 / 10$ & $0.079^{\mathrm{a}}$ \\
\hline Age (years) & $52.16 \pm 8.68$ & $53.11 \pm 7.87$ & $53.87 \pm 7.50$ & $53.67 \pm 7.86$ & $0.861^{\mathrm{b}}$ \\
\hline Body weight (kg) & $60.00 \pm 9.25$ & $64.18 \pm 7.42$ & $64.20 \pm 10.05$ & $62.87 \pm 7.00$ & $0.289^{\mathrm{b}}$ \\
\hline Body height (m) & $1.62 \pm 0.067$ & $1.66 \pm 0.065$ & $1.63 \pm 0.065$ & $1.64 \pm 0.067$ & $0.160^{\mathrm{b}}$ \\
\hline BMI (kg/m²) & $22.83 \pm 3.06$ & $23.13 \pm 1.44$ & $24.18 \pm 3.09$ & $23.44 \pm 2.48$ & $0.142^{\mathrm{b}}$ \\
\hline SS (degrees) & $29.4(25.6,33.5)$ & $31.8(28.8,34.3)$ & $39.0(37.2,41.0)$ & $45.9(45.2,48.3)$ & $<0.001^{\mathrm{c}}$ \\
\hline PI (degrees) & $39.1 \pm 5.6$ & $41.1 \pm 5.5$ & $49.8 \pm 8.2$ & $58.4 \pm 7.3$ & $<0.001^{\mathrm{b}}$ \\
\hline PT (degrees) & $9.9 \pm 5.0$ & $10.0 \pm 4.5$ & $10.8 \pm 7.3$ & $11.4 \pm 5.2$ & $0.210^{\mathrm{b}}$ \\
\hline LL (degrees) & $38.5(31.9,46.1)$ & $44.5(35.7,48.8)$ & $53.2(47.7,56.5)$ & $63.8(60.0,68.7)$ & $<0.001^{\mathrm{c}}$ \\
\hline
\end{tabular}

Values are expressed as $\mathrm{n}(\%)$, the mean \pm standard deviation or median (interquartile range). Statistical analysis of baseline information and spinopelvic parameters in different lumbar spinal subgroups: ${ }^{a} \chi^{2}$ test, ${ }^{b}$ one-way analysis of variance, ${ }^{c}$ Kruskal-Wallis test. BMI, body mass index; SS, sacral slope; PI, pelvic incidence; PT, pelvic tilt; LL, lumbar lordosis.
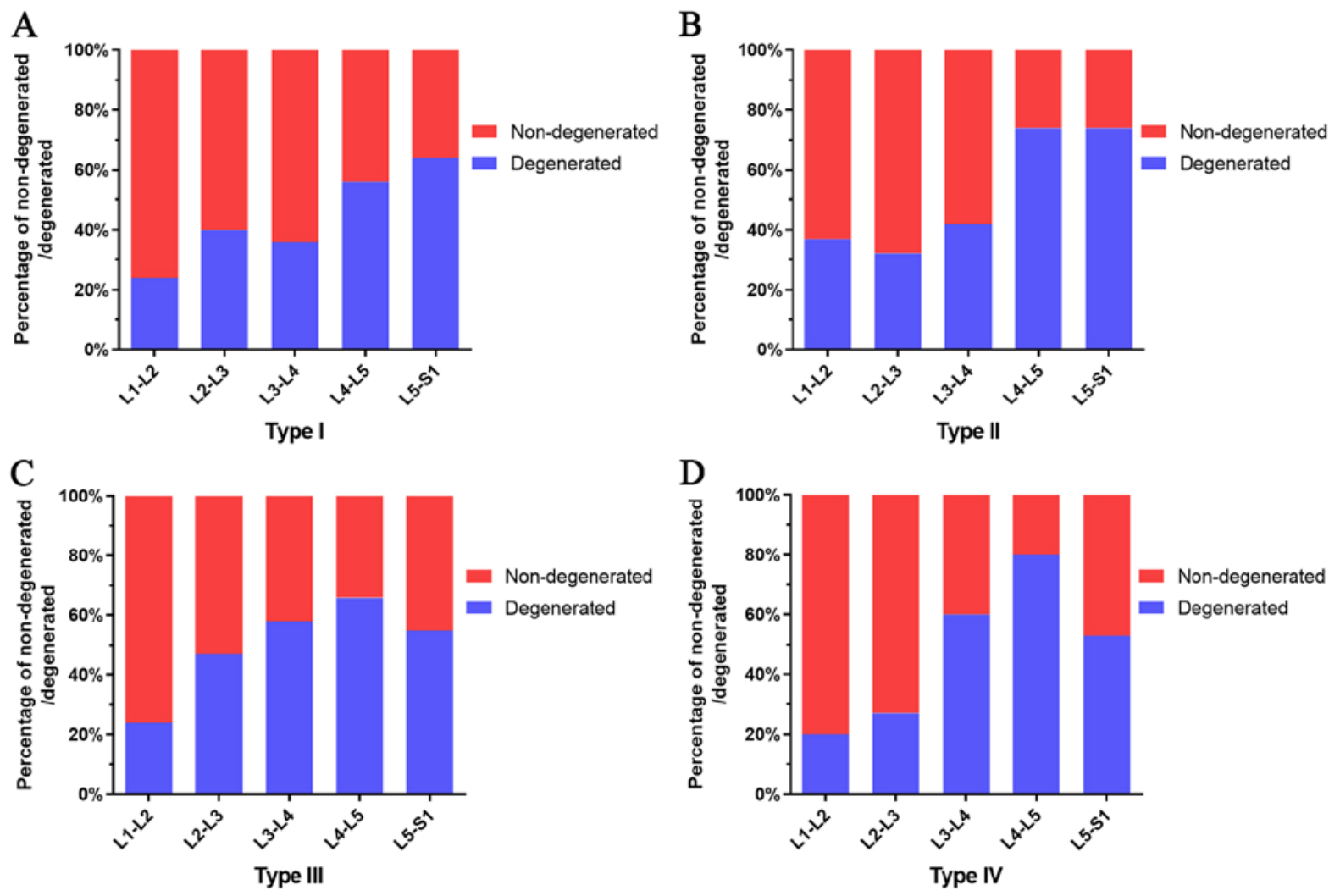

Figure 4. Proportion of disc degeneration and non-degeneration by level in four lumbar spinal subgroups. (A) Type I, (B) Type II, (C) Type III and (D) Type IV.

according to the Pfirrmann classification. Among each LSS, the distribution of the degree of the LDD at each sequential lumbar level from L1/L2 to L5/S1 is presented in Table II. Across all LSS groups, L1/L2, L2/L3 and L3/L4, LDD was mainly indicated to be grade II and III, and L4/L5, L5/S1 was mainly indicated to be grade III and IV, but grade I and V were rarely observed at all levels. Furthermore, despite the different subgroups, the proportion of disc degeneration increased from proximal toward distal (Table III and Fig. 4). The percentage of degenerated discs in groups I-IV based on the Roussouly classification was $\sim 50 \%$, accounting for $44,52,50$ and $48 \%$, respectively (Table III).

No differences among LSS. Based on the evaluation of the proportion of disc degeneration at each level, no statistically significant difference among types I to IV was indicated (Table III). LDD was not indicated to be significantly associated with lumbar spinal morphology, nor was higher spinopelvic parameters protective against LDD among asymptomatic middle-aged and aged adults (Table III). 
Table II. Distribution of the lumbar disc degeneration degree in each lumbar spinal subgroup.

A, Roussouly type I

\begin{tabular}{lrrrrr}
\hline Pfirrmann classification & L1-L2 & L2-L3 & L3-L4 & L4-L5 \\
\hline I & 3 & 0 & 1 & 0 & 0 \\
II & 16 & 15 & 15 & 11 & 8 \\
III & 5 & 5 & 7 & 5 \\
IV & 1 & 4 & 2 & 6 \\
V & 0 & 1 & 0 & 0 & 1 \\
\hline
\end{tabular}

B, Roussouly type II

\begin{tabular}{lrrrrr}
\hline Pfirrmann classification & L1-L2 & L2-L3 & L3-L4 & L4-L5 & L5-S1 \\
\hline I & 0 & 0 & 1 & 0 & 0 \\
II & 12 & 13 & 10 & 5 & 5 \\
III & 5 & 3 & 5 & 4 & 5 \\
IV & 1 & 3 & 3 & 9 & 6 \\
V & 1 & 0 & 0 & 1 & 3
\end{tabular}

C, Roussouly type III

\begin{tabular}{lrrrrr}
\hline Pfirrmann classification & L1-L2 & L2-L3 & L3-L4 & L4-L5 & L5-S1 \\
\hline I & 0 & 1 & 1 & 1 & 4 \\
II & 29 & 19 & 14 & 12 & 13 \\
III & 7 & 16 & 13 & 13 & 4 \\
IV & 2 & 2 & 9 & 1 & 16 \\
V & 0 & 0 & 1 & 1 \\
\hline
\end{tabular}

D, Roussouly type IV

\begin{tabular}{lrcccc}
\hline Pfirrmann classification & L1-L2 & L2-L3 & L3-L4 & L4-L5 & L5-S1 \\
\hline I & 0 & 0 & 0 & 2 & 2 \\
II & 12 & 11 & 6 & 1 & 5 \\
III & 3 & 4 & 7 & 4 & 1 \\
IV & 0 & 0 & 2 & 0 & 7 \\
V & 0 & 0 & 0 & & 0 \\
\hline
\end{tabular}

\section{Discussion}

The present study included a total of 97 volunteers aged $>40$ years and investigated the effect of LSS on the prevalence of lumbar intervertebral disc degeneration. The prevalence of LDD at the caudal lumbar intervertebral levels at L4/5 and L5/S1 was significantly higher compared with the proximal levels in all types. Contact forces and shear stress primarily act on the caudal region of the lumbar spine due to body weight stress (19); therefore, the increased mechanical stress at L4/5 and L5/S1 may lead to the pathological procress of degeneration.

Furthermore, it was demonstrated that the frequency of disc degeneration from type I to IV was 44, 52, 50 and 48\%, respectively, in a population of middle-aged and aged asymptomatic subjects, which was significantly higher compared with that in young individuals in a previous study (4). It was clear that age was an important and non-negligible factor that contributed to the increase of LDD. For instance, disc degeneration in $88 \%$ of individuals aged $>55$ years was increased compared with $42 \%$ in subjects aged $<30$ years $(20)$.

The pelvic position and shape interact with the spinal organization and regulate the balance between the spine and pelvis. According to biomechanical analysis, spinopelvic sagittal alignment was expected to explain intervertebral disc degeneration. Therefore, with regard to spinopelvic parameters, Roussouly and Pinheiro-Franco (14) categorized LSS as type I to IV, which are all considered to be normal. Excluding extrinsic triggers of intervertebral disc degeneration, including 


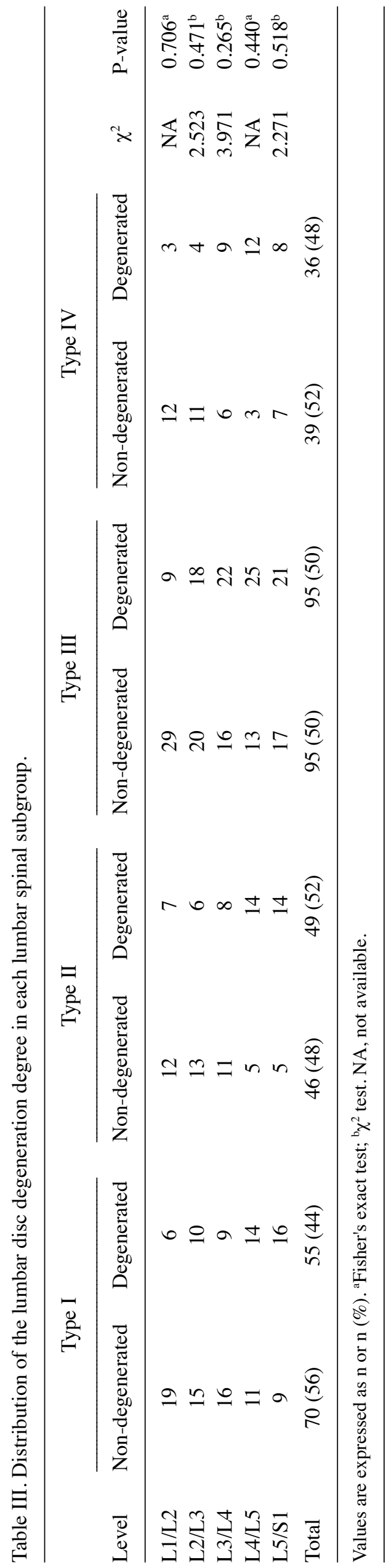

physical activity $(21,22)$, the parameters of lumbar spine sagittal alignment, including LL and PI, were considered to be strong predictors and serve a predisposing role in the pathogenesis of LDD diseases $(12,23)$. SS, PI and LL have been observed to be different from type I to IV $(5,23)$, thus, it was possible that different LSS (I-IV) may influence level-specific degeneration via specific biomechanical stressors. The Roussouly type II subtype is a flat lordosis that is characterized by mild thoracic and lumbar curvatures, with an SS of $<35^{\circ}$. Therefore, stress is at its maximum on the discs in type II, and therefore, this type is associated with a higher risk of disc degeneration compared with type IV. Although the higher prevalence of lower back pain has been demonstrated in the type II subtype (14), the impact of LSS on the degeneration of the intervertebral disc has remained to be identified. A number of studies have refuted the theory regarding the influence of the spinal structure on the progress of LDD. Battie et al (24) reported that heredity, as opposed to physical loading, has a relatively dominant role in the progress of disc degeneration, which may explain the high prevalence of up to $74 \%$ seen in the general population. Torrie and Videman (5) suggested that LSS was not statistically associated with LDD and a higher PI was not a protective factor against LDD. Similarly, in the present study, the difference of spinopelvic parameters was observed, including SS, PI and LL, but level-specific degeneration was indicated to not be significantly different among LSS.

Previous studies on the effect of genetic factors on the LDD process also supported the influence of genetic defects on the structural and functional changes in the intervertebral disc, which may compromise the disc's mechanical properties and metabolic activities (9,24-27). Battie et al (24) considered that genetic factors and not physical loading specific to occupation and sport served the dominant role in disc degeneration in a previous study involving twins. Based on the present results, it was hypothesized that genetic heritability and not spinal biomechanical differences result in the initialization of LDD in a specific age group, particularly in older populations. Heritability has previously been described as the proportion of phenotypic variation within a population, where the trait of disc degeneration has been suggested to be heritable in a previous study (28). Genetic factors may become major risk factors and contribute to the patho-etiology of LDD, as described by various studies $(3,24,29)$. In a previous study on spines of twins, genetic heritability was demonstrated to be associated with lower back pain (3). Recently, a review also highlighted the genetic basis of LDD and assessed how genetic variants influenced IDD using cell biology (29).

The present study was not the first to identify the association between lumbar spine sagittal alignment and disc degeneration in an asymptomatic population. A previous study reported that the subtype II was significantly associated with disc degeneration at L4-L5 in asymptomatic young adults (4), which was in contradiction with the results of the present study. It may be suggested that genetic heritability may be a major factor in the process of intervertebral disc degeneration in older individuals and lumbar spine sagittal alignment may not influence disc degeneration. Genes were not indicated to exhibit the power to cause disc degeneration and this may have been the reason why the high prevalence was reduced to $42 \%$ in the young population. Therefore, biomechanical factors may have a weak role at one specific level. Future studies 
should focus on biomechanical factors in the young asymptomatic population and increase attention to other factors in the older population. Regarding the clinical prevention of intervertebral disc degeneration, the present study indicated that it may not be necessary for spinal surgeons to evaluate LSS in asymptomatic middle-aged and aged individuals.

A number of limitations were present in the current study. First, genetic factors were not assessed, which may be a major risk factor for this disorder. This was why only the biomechanical factors were evaluated. Furthermore, the small number of asymptomatic volunteers included in the present study may result in a large error. In future studies, it is essential to assess more volunteers in a larger cohort study. Finally, the study was retrospective and was not a longitudinal cohort study. However, the present study successfully demonstrated that structural differences are not a risk factor in LSS and did not influence LDD in the study on subjects aged $>40$ years.

In conclusion, in the present study, no correlation between LSS and intervertebral disc degeneration was observed among asymptomatic middle-aged and aged subjects. In addition, other risk factors may serve a vital role in disc degeneration in asymptomatic individuals of these age groups and this requires further study.

\section{Acknowledgements}

Not applicable.

\section{Funding}

The current study was supported by grants from the National Nature Foundation of China (grant no. 81871806) and the Zhejiang Public Service Technology Research Program/Social Development (grant no. LGF18H060008).

\section{Availability of data and materials}

The datasets used and/or analyzed during the current study are available from the corresponding author on reasonable request.

\section{Authors' contributions}

BW, YZY and XYW designed the study. BW, JLL, LJC, YZY, $\mathrm{XYW}$ and YFS recruited volunteers. BW, CAH, YZY, XYW and YFS collected the data. BW, BDC and ZXS analyzed the data. CAH, YZY and XYW performed the radiological analysis and interpreted the results. BW, LJC and ZXS organized the manuscript. JLL reviewed the papers and revised the manuscript. All authors have read and approved the final version of the manuscript. All authors contributed toward data analysis, drafting and revising the paper and agree to be accountable for all aspects of the work.

\section{Ethics approval and consent to participate}

The present study was approved by the Ethics Board of the Second Affiliated Hospital and Yuying Children's Hospital of Wenzhou Medical University (2016 number 10; Wenzhou, China). Written informed consent for publication was obtained from all individual participants included in the study.

\section{Patient consent for publication}

Not applicable.

\section{Competing interests}

The authors declare that they have no competing interests.

\section{References}

1. Luoma K, Riihimaki H, Luukkonen R, Raininko $\mathrm{R}$, Viikari-Juntura $\mathrm{E}$ and Lamminen A: Low back pain in relation to lumbar disc degeneration. Spine (Phila Pa 1976) 25: 487-492, 2000.

2. Murray CJ, Vos T, Lozano R, Naghavi M, Flaxman AD, Michaud C, Ezzati M, Shibuya K, Salomon JA, Abdalla S, et al: Disability-adjusted life years (DALYs) for 291 diseases and injuries in 21 regions, 1990-2010: A systematic analysis for the Global Burden of Disease Study 2010. Lancet 380: 2197-2223, 2012.

3. Livshits G, Popham M, Malkin I, Sambrook PN, Macgregor AJ, Spector T and Williams FM: Lumbar disc degeneration and genetic factors are the main risk factors for low back pain in women: The UK Twin Spine Study. Ann Rheum Dis 70: 1740-1745, 2011.

4. Menezes-Reis R, Bonugli GP, Dalto VF, da Silva Herrero CF, Defino HL and Nogueira-Barbosa MH: Association between lumbar spine sagittal alignment and L4-L5 Disc degeneration among asymptomatic young adults. Spine (Phila Pa 1976) 41: E1081-E1087, 2016.

5. Torrie PA, McKay G, Byrne R, Morris SA and Harding IJ: The influence of lumbar spinal subtype on lumbar intervertebral disc degeneration in young and Middle-aged adults. Spine Deform 3: 172-179, 2015.

6. Battie MC, Videman T, Gill K, Moneta GB, Nyman R, Kaprio J and Koskenvuo M: 1991 Volvo Award in clinical sciences. Smoking and lumbar intervertebral disc degeneration: An MRI study of identical twins. Spine (Phila Pa 1976) 16: 1015-1021, 1991.

7. Hangai M, Kaneoka K, Kuno S, Hinotsu S, Sakane M, Mamizuka N, Sakai S and Ochiai N: Factors associated with lumbar intervertebral disc degeneration in the elderly. Spine J 8: 732-740, 2008.

8. Leino-Arjas P, Kaila-Kangas L, Solovieva S, Riihimaki H, Kirjonen J and Reunanen A: Serum lipids and low back pain: An association? A follow-up study of a working population sample. Spine (Phila Pa 1976) 31: 1032-1037, 2006.

9. Feng Y, Egan B and Wang J: Genetic factors in intervertebral disc degeneration. Genes Dis 3: 178-185, 2016.

10. Xia DD, Lin SL, Wang XY, Wang YL, Xu HM, Zhou F and Tan J: Effects of shear force on intervertebral disc: An in vivo rabbit study. Eur Spine J 24: 1711-1719, 2015.

11. Vergroesen PP, Kingma I, Emanuel KS, Hoogendoorn RJ, Welting TJ, van Royen BJ, van Dieën JH and Smit TH: Mechanics and biology in intervertebral disc degeneration: A vicious circle. Osteoarthritis Cartilage 23: 1057-1070, 2015.

12. Ergun T, Lakadamyali $\mathrm{H}$ and Sahin MS: The relation between sagittal morphology of the lumbosacral spine and the degree of lumbar intervertebral disc degeneration. Acta Orthop Traumatol Turc 44: 293-299, 2010.

13. Roussouly P, Gollogly S, Berthonnaud E and Dimnet J: Classification of the normal variation in the sagittal alignment of the human lumbar spine and pelvis in the standing position. Spine (Phila Pa 1976) 30: 346-353, 2005.

14. Roussouly P and Pinheiro-Franco JL: Biomechanical analysis of the spino-pelvic organization and adaptation in pathology. Eur Spine J 20 (Suppl 5): S609-S618, 2011.

15. Yan YZ, Shao ZX, Pan XX, Chen SQ, Wu AM, Tian NF, Wu YS and Wang XY: Acceptable Chin-brow vertical angle for neutral position radiography: Preliminary analyses based on parameters of the whole sagittal spine of an asymptomatic chinese population. World Neurosurg 120: e488-e496, 2018.

16. Glassman SD, Berven S, Bridwell K, Horton $\mathrm{W}$ and Dimar JR: Correlation of radiographic parameters and clinical symptoms in adult scoliosis. Spine (Phila Pa 1976) 30: 682-688, 2005. 
17. Aota Y, Saito T, Uesugi M, Ishida K, Shinoda K and Mizuma K: Does the fists-on-clavicles position represent a functional standing position? Spine (Phila Pa 1976) 34: 808-812, 2009.

18. Pfirrmann CW, Metzdorf A, Zanetti M, Hodler J and Boos N Magnetic resonance classification of lumbar intervertebral disc degeneration. Spine (Phila Pa 1976) 26: 1873-1878, 2001.

19. Keller TS, Colloca CJ, Harrison DE, Harrison DD and Janik TJ Influence of spine morphology on intervertebral disc loads and stresses in asymptomatic adults: Implications for the ideal spine. Spine J 5: 297-309, 2005.

20. Cheung KM, Chan D, Karppinen J, Chen Y, Jim JJ, Yip SP, Ott J, Wong KK, Sham P, Luk KD, et al: Association of the Taq I allele in vitamin $\mathrm{D}$ receptor with degenerative disc disease and disc bulge in a Chinese population. Spine (Phila Pa 1976) 31: 1143-1148, 2006.

21. Elfering A, Semmer N, Birkhofer D, Zanetti M, Hodler J and Boos N: Risk factors for lumbar disc degeneration: A 5-year prospective MRI study in asymptomatic individuals. Spine (Phila Pa 1976) 27: 125-134, 2002.

22. Samartzis D, Karppinen J, MokF, Fong DY, Luk KD and Cheung KM: A population-based study of juvenile disc degeneration and its association with overweight and obesity, low back pain, and diminished functional status. J Bone Joint Surg Am 93: 662-670, 2011.

23. Yang X, Kong Q, Song Y, Liu L, Zeng J and Xing R: The characteristics of spinopelvic sagittal alignment in patients with lumbar disc degenerative diseases. Eur Spine J 23: 569-575, 2014.
24. Battie MC and Videman T: Lumbar disc degeneration: Epidemiology and genetics. J Bone Joint Surg Am 88 (Suppl 2): S3-S9, 2006.

25. Chan D, Song Y, Sham P and Cheung KM: Genetics of disc degeneration. Eur Spine J 15 (Suppl 3): S317-S325, 2006.

26. Cheung KM: The relationship between disc degeneration, low back pain, and human pain genetics. Spine J 10: 958-960, 2010.

27. Kalichman $L$ and Hunter DJ: The genetics of intervertebral disc degeneration. Associated genes. Joint Bone Spine 75: 388-396, 2008

28. Williams FM, Popham M, Sambrook PN, Jones AF, Spector TD and MacGregor AJ: Progression of lumbar disc degeneration over a decade: A heritability study. Ann Rheum Dis 70: 1203-1207, 2011.

29. Munir S, Rade M, Maatta JH, Freidin MB and Williams FMK: Intervertebral disc biology: Genetic basis of disc degeneration. Curr Mol Biol Rep 4: 143-150, 2018.

cc (i) $(\Theta$ This work is licensed under a Creative Common Attribution-NonCommercial-NoDerivatives 4.0 International (CC BY-NC-ND 4.0) License. 